\title{
A Case of Pancreatic Carcinoma in situ Diagnosed by Repeated Pancreatic Juice Cytology
}

\author{
Takeshi Miyata Mamoru Takenaka Shunsuke Omoto Ken Kamata \\ Kosuke Minaga Kentaro Yamao Hajime Imai Masatoshi Kudo \\ Department of Gastroenterology and Hepatology, Kindai University Faculty of Medicine, Osaka-Sayama, Japan
}

\section{Keywords}

Pancreatic carcinoma in situ · Pancreatic juice cytology

\begin{abstract}
Repeated pancreatic juice cytology via endoscopic nasopancreatic drainage (ENPD) has a high diagnostic yield and might be useful for the diagnosis of early-stage pancreatic cancer. A 67-year-old man presented with a pancreatic cyst occasionally detectable in the body of the pancreas by ultrasonography (US). No obvious pancreatic tumor was detected by US, computed tomography (CT), magnetic resonance cholangiopancreatography, and endoscopic ultrasound (EUS) (although the latter did reveal a weak, low echoic area). Endoscopic retrograde pancreatography showed irregular narrowing of the main pancreatic duct (MPD) at the pancreatic body. Pancreatic juice cytology was also performed, but did not give evidence of a malignancy. Therefore, the patient was followed up. CT and EUS performed after 3 months showed the same findings as did endoscopic retrograde pancreatography; however, the results of repeated pancreatic juice cytology performed via ENPD tube revealed a suspected malignancy on 2 of 6 occasions. Therefore, we performed a central pancreatectomy. Histopathological exami-
\end{abstract}

\section{KARGER}

(C) 2017 S. Karger AG, Basel

E-Mail karger@karger.com

www.karger.com/ocl nation of a resected specimen revealed carcinoma in situ in the narrow MPD at the body of the pancreas. In the current case, repeated pancreatic juice cytology via ENPD was effective. A weak low echoic area around the MPD stricture on EUS might be related to the inflammatory change accompanying carcinoma in situ of the pancreas.

(c) 2017 S. Karger AG, Basel

\section{Introduction}

Repeated pancreatic juice cytology via endoscopic nasopancreatic drainage (ENPD) is found to have a high diagnostic yield and might be useful for the diagnosis of early-stage pancreatic cancer [1]. Endoscopic ultrasound (EUS)-guided fine-needle aspiration is also helpful for confirming the diagnosis of early pancreatic carcinoma, but its role is limited with respect to carcinoma in situ as no tumor has formed at this stage [2]. A weak low echoic area around the main pancreatic duct (MPD) stricture on EUS might be related to inflammatory changes accompanying carcinoma in situ of the pancreas.

Here, we report the case of repeated pancreatic juice cytology via ENPD, which was effective.

Dr. Mamoru Takenaka

Department of Gastroenterology and Hepatology Kindai University Faculty of Medicine

377-2 Ohno-Higashi, Osaka-Sayama, Osaka 589-8511 (Japan)

E-Mailmamoxyo45@gmail.com 


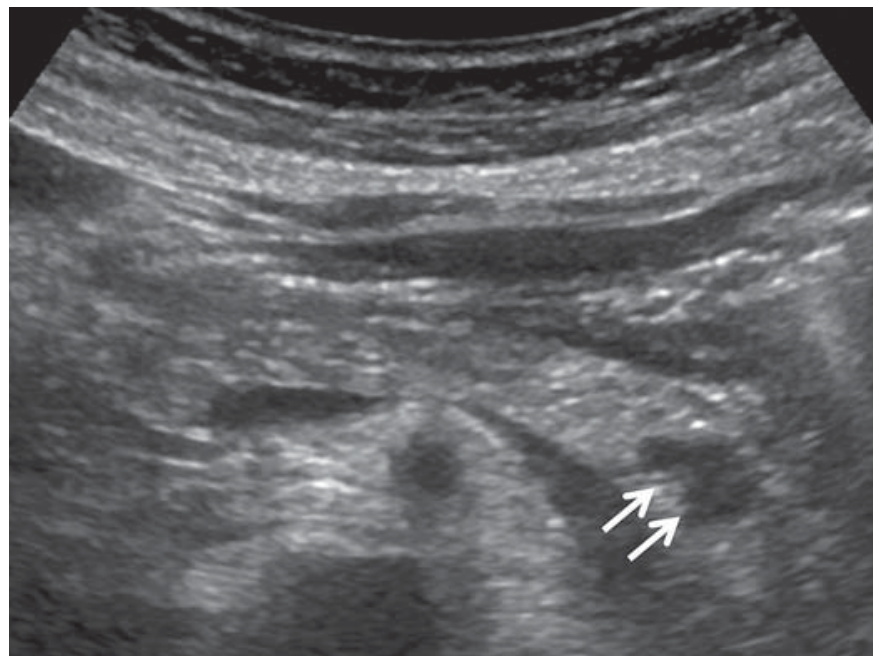

Fig. 1. An ultrasonography image showing a pancreatic cyst in the body of the pancreas (arrows).

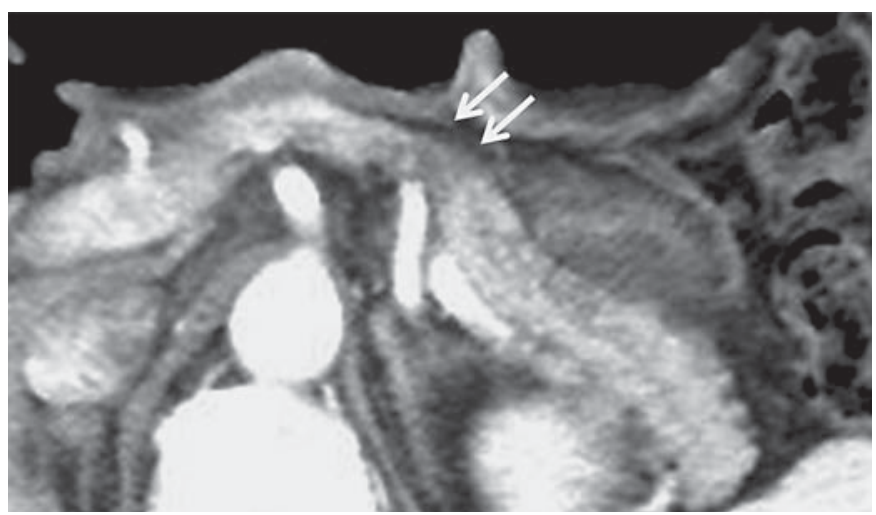

Fig. 2. A computed tomography image showing a pancreatic cyst in the body, and dilation of the main pancreatic duct (arrows).

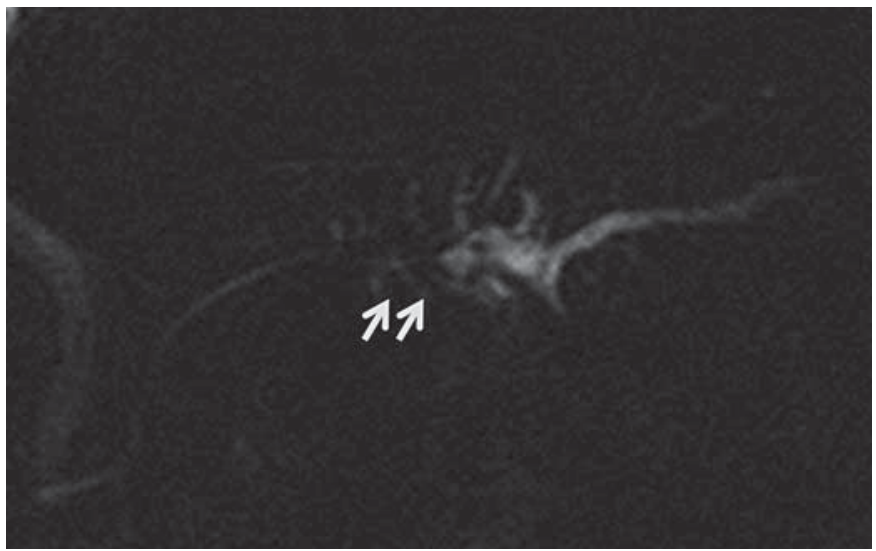

Fig. 3. A magnetic resonance cholangiopancreatography image showing irregular narrowing of the main pancreatic duct at the pancreatic body (arrows).

Pancreatic Carcinoma in situ Diagnosed by Pancreatic Juice Cytology

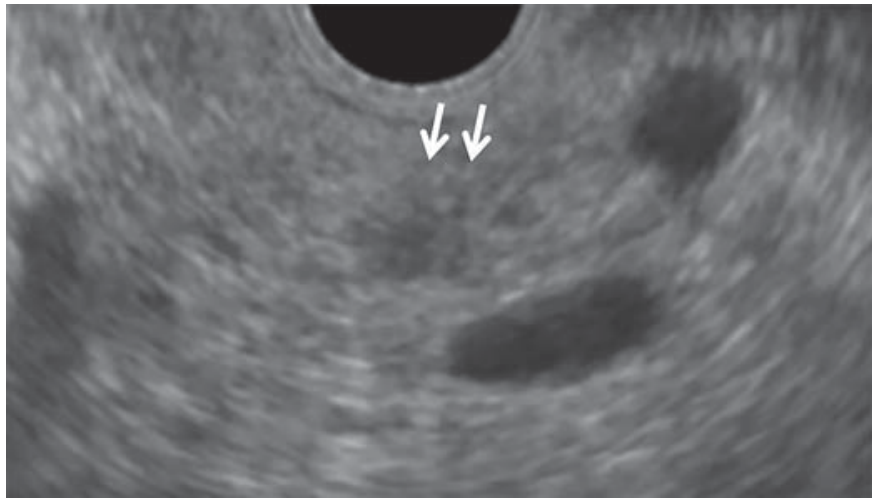

Fig. 4. An endoscopic ultrasound image showing a weak low echoic area around the narrowing of the main pancreatic duct at the pancreatic body (arrows).

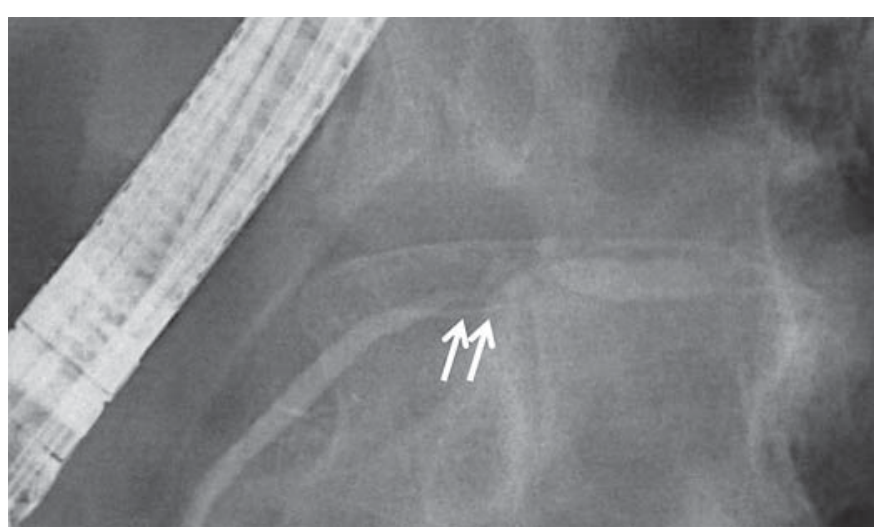

Fig. 5. An endoscopic retrograde pancreatography image showing irregular narrowing of the main pancreatic duct at the pancreatic body (arrows).

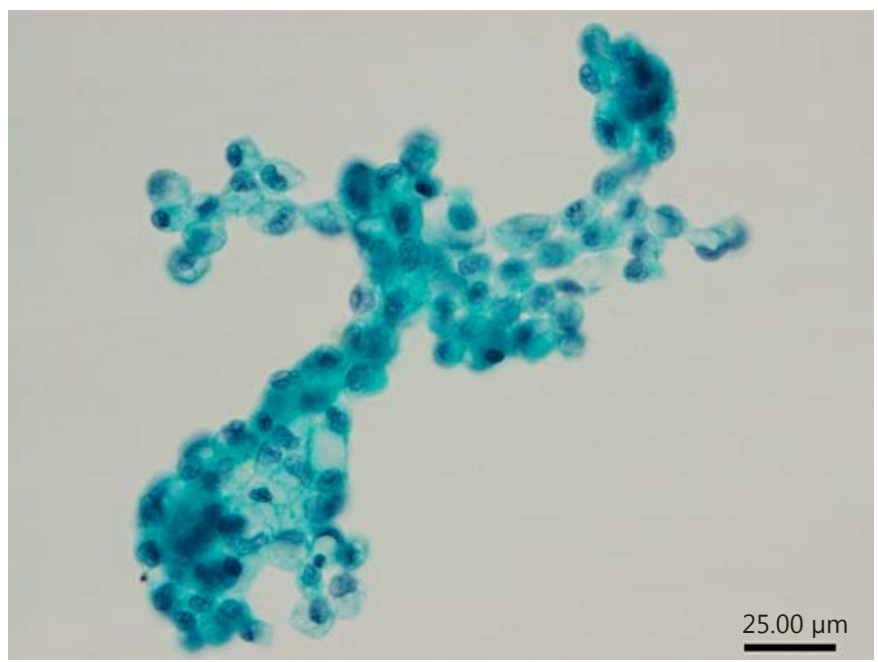

Fig. 6. Pancreatic juice cytology obtained by endoscopic nasopancreatic drainage tube revealing suspected malignancy.

Oncology 2017;93(suppl 1):98-101 
Fig. 7. Histopathological examination of the resected specimen shows carcinoma in situ at the part of the narrow main pancreatic duct in the body of the pancreas (arrows).
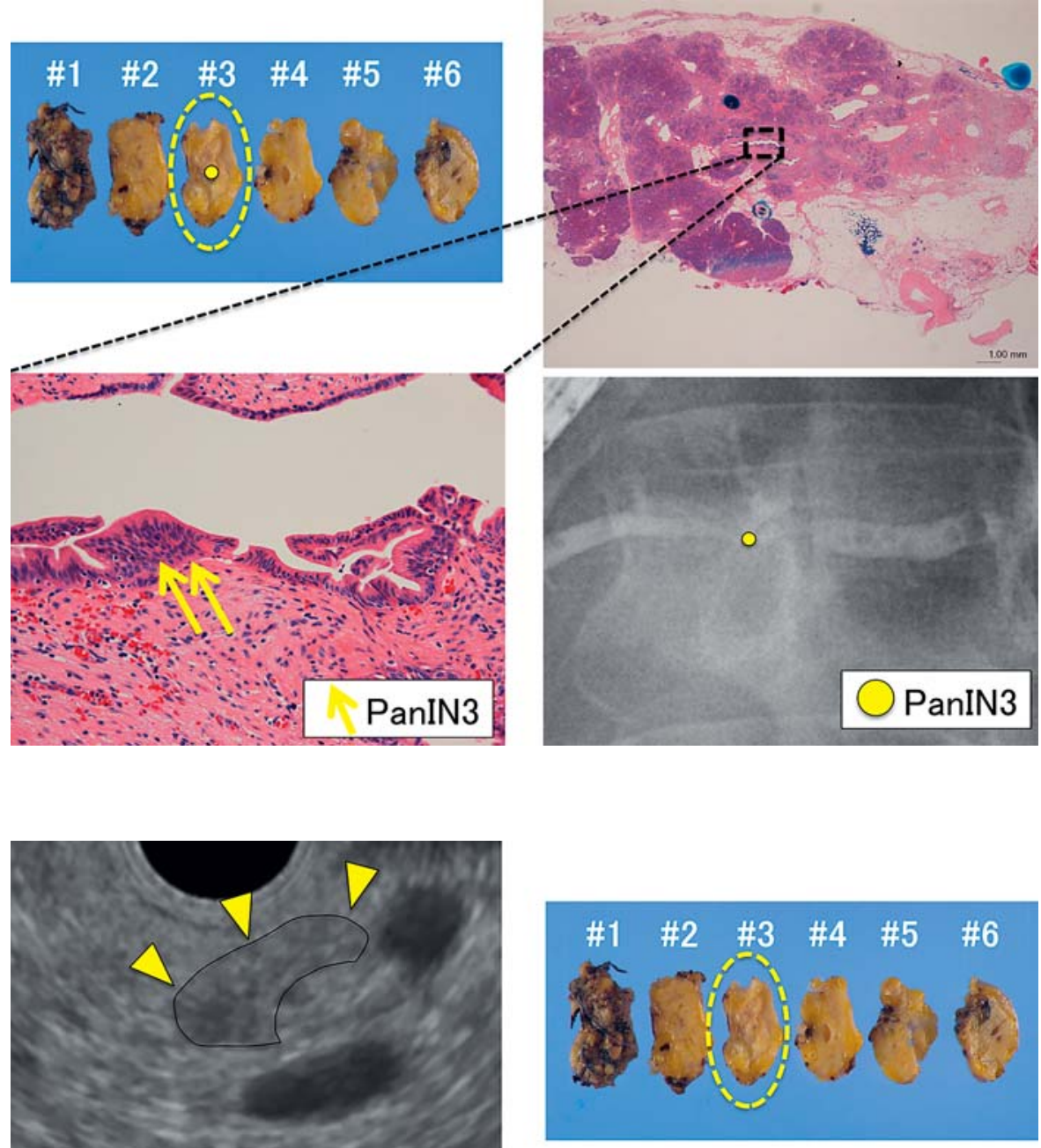

Fig. 8. Histopathological examination of the resected specimen shows inflammatory change seen around the carcinoma (arrows) in situ detected by endoscopic ultrasound (arrowheads).

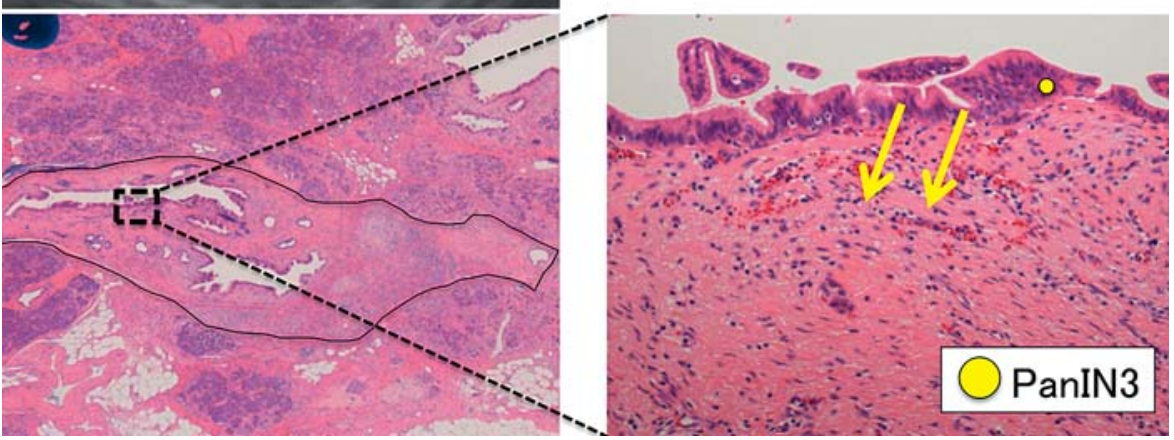

\section{Case Report}

We describe a 67-year-old man with a pancreatic cyst occasionally detectable in the body of the pancreas by ultrasonography (US) (Fig. 1). Tumor markers (CEA, CA19-9, DUPAN2, and Span1) were within normal limits. Computed tomography (CT) also revealed a pancreatic cyst (diameter, $15 \mathrm{~mm}$ ) in the body of the pancreas and dilation of the MPD (Fig. 2). Magnetic resonance cholangiopancreatography showed localized irregular narrowing of the MPD at the pancreatic body (Fig. 3), and EUS showed a weak low echoic area around this point (Fig. 4). No obvious pancreatic tumor was detected by US, CT, magnetic resonance cholangiopancreatography, and EUS (although the latter did reveal a weak low echoic area). Endoscopic retrograde pancreatography 
showed irregular narrowing of the MPD at the pancreatic body (Fig. 5). Pancreatic juice cytology was also performed, but did not give evidence of a malignancy. Therefore, the patient was followed up. CT and EUS performed 3 months later showed the same findings as did endoscopic retrograde pancreatography; however, the results of repeated pancreatic juice cytology via ENPD tube revealed a suspected malignancy on 2 of 6 occasions (Fig. 6). Taking all imaging findings into consideration, we suspected pancreatic carcinoma in situ at the pancreatic body. Therefore, we performed a central pancreatectomy. Histopathological examination of a resected specimen revealed carcinoma in situ in the narrow MPD at the body of the pancreas (Fig. 7). The resected margin of the pancreas was negative, and no lymph node metastasis was detected. Inflammatory change (inflammatory cell infiltration and fibrosis) were observed around the carcinoma in situ, at the site matched to the low echoic area detected by EUS (Fig. 8). The patient is still alive 3 years following surgery, and no adjuvant chemotherapy was required.

\section{Discussion}

Previous studies showed that ENPD had a high diagnostic yield and might be useful for the diagnosis of earlystage pancreatic cancer [1]. In the current case, repeated

pancreatic juice cytology via ENPD was also effective. EUS-guided fine-needle aspiration is also helpful for confirming the diagnosis of early pancreatic carcinoma, but its role is limited with respect to carcinoma in situ as no tumor has formed at this stage [2]. A weak low echoic area around the MPD stricture on EUS might be related to the inflammatory changes accompanying carcinoma in situ of the pancreas.

\section{Disclosure Statement}

The authors have no conflicts of interest to declare.

\section{References}

1 Mikata R, Ishihara T, Yokosuka O, et al: Clinical usefulness of repeated pancreatic juice cytology via endoscopic naso-pancreatic drainage tube in patients with pancreatic cancer. J Gastroenterol 2013;48:866-873.

2 Hanada K, Okazaki A, Yonehara S, et al: Diagnostic strategies for early pancreatic cancer. J Gastroenterol 2015;50:147-154. 